\title{
Blood pressure in postmenopausal women with a history of polycystic ovary syndrome
}

\author{
Katarzyna Doroszewska1, Tomasz Milewicz' ${ }^{1}$, Sandra Mrozińska², Jarosław Janeczko³, Radosław Rokicki \\ Marek Janeczko3, Damian Warzecha ${ }^{4}$, Piotr Marianowski ${ }^{4}$
}

${ }^{1}$ Department of Gynaecological Endocrinology and Gynaecology, Jagiellonian University Medical College, Cracow, Poland ${ }^{2}$ Department of Metabolic Diseases, Jagiellonian University Medical College, Cracow, Poland

${ }^{3}$ Infertility Treatment Centre PARENS, Cracow, Poland

${ }^{4} 1^{\text {st }}$ Department of Obstetrics and Gynaecology, Medical University of Warsaw, Warsaw, Poland

\begin{abstract}
Polycystic ovarian syndrome (PCOS) is the most common endocrine disorder at reproductive age, affecting $6-10 \%$ of females in this group. The aetiology of this syndrome is not fully understood. Genetics, endocrinology factors, and the influence of the environment are possible causes of this syndrome. PCOS is characterised by menstrual disorders, hyperandrogenism, and abnormalities in ovarian morphology as well as metabolic disorders. PCOS increases the risk of overweight and obesity, diabetes, endometrial cancer, and cardiovascular diseases such as hypertension along with all its long-term consequences. There are limited studies about cardiovascular disorders, especially hypertension, in postmenopausal women with a history of PCOS. The presented paper is an attempt to briefly summarise literature data concerning the influence of this disease on the incidence of hypertension and blood pressure control in postmenopausal women. Women with PCOS more often present features of metabolic syndrome and have increased cardiovascular risk factors including hypertension. The prevalence of hypertension is 2.5 times higher than in corresponding healthy peers. Furthermore, hyperandrogenaemia is associated with elevated blood pressure independent of the patient's age, insulin resistance, obesity, and dyslipidaemia. In view of this, these patients should be thoroughly screened for hypertensive disorders and educated about the lifestyle modifications that could prevent hypertension later in life.
\end{abstract}

Key words: polycystic ovary syndrome, menopause, blood pressure, hyperandrogenaemia, hypertension.

\section{Introduction}

Polycystic ovary syndrome (PCOS) is one of the commonest female endocrinopathies. PCOS is a highly prevalent disorder affecting about $6-10 \%$ of women of reproductive age [1-3] and has long-term fallout that goes beyond reproductive age [4]. Its aetiopathogenesis is complex, multifactorial, and heterogeneous, including the interaction of genetic, epigenetic, and environmental factors [5]. The common clinical manifestations of this syndrome are oligo-anovulation, hyperandrogenism, and/or hyperandrogenaemia and polycystic ovary morphology $[2,6,7]$. PCOS is established as a metabolic disorder [6]. Cardiovascular risk factors such as hypertension, dyslipidaemia, metabolic syndrome, and obesity are more frequent among women with PCOS compared to non-PCOS women $[6,9]$. This has led to the assumption that women with PCOS have a higher risk of developing cardiovascular diseases in later life $[6,10,11]$.

There are limited studies about cardiovascular disorders, especially hypertension, in postmenopausal women with a history of PCOS. Most authors suggest that PCOS in postmenopausal women increases the risk of hypertension with all its consequences.

\section{Material and methods}

The authors searched the PubMed database with different combinations of the key words: "polycystic ovary syndrome", "menopause", "blood pressure", and "hypertension". The established inclusion criteria for the following review were: an original prospective, cross-sectional or retrospective study with at least five years of follow-up, and a study group of at least 30 participants. Only six trials met the inclusion criteria. All of them are summarised in Table 1. 
Table 1. Studies on cardiovascular disease risk in women with polycystic ovarian syndrome (PCOS)

\begin{tabular}{lcccl}
\hline Study & Type of study & $\begin{array}{c}\text { PCOS } \\
\text { vs. control }(n)\end{array}$ & $\begin{array}{c}\text { Mean or median age } \\
\text { at follow-up (years) }\end{array}$ & \multicolumn{1}{c}{ Conclusions } \\
\hline $\begin{array}{l}\text { Dahlgren [17] } \\
1992\end{array}$ & Cross-sectional & 33 vs. 132 & $40-59$ & $\begin{array}{l}\text { Perimenopausal women with PCOS have increased } \\
\text { morbidity in hypertension and diabetes mellitus }\end{array}$ \\
\hline $\begin{array}{l}\text { Wild [16] } \\
2000\end{array}$ & Retrospective & 319 vs. 1060 & $\begin{array}{c}56.7 \text { (31 years } \\
\text { follow up) }\end{array}$ & $\begin{array}{l}\text { Increased prevalence of hypertension in } \\
\text { menopausal-PCOS women compared to a cohort } \\
\text { of control women }\end{array}$ \\
\hline $\begin{array}{l}\text { Elting [15] } \\
2001\end{array}$ & Retrospective & 32 vs. 2372 & $45-54$ & $\begin{array}{l}\text { 2.5-times higher prevalence of hypertension than } \\
\text { the corresponding age group of the Dutch female } \\
\text { population }\end{array}$ \\
\hline $\begin{array}{l}\text { Schmidt [12] } \\
2011\end{array}$ & Cross-sectional & 32 vs. 120 & $\begin{array}{l}61-79 \\
\text { (after 21 years } \\
\text { of follow up) }\end{array}$ & $\begin{array}{l}\text { High prevalence of hypertension and high } \\
\text { triglyceride levels in postmenopausal PCOS } \\
\text { women }\end{array}$ \\
\hline $\begin{array}{l}\text { Armeni [13] } \\
2013\end{array}$ & Cross-sectional & 43 vs. 286 & $55.6 \pm 7.8$ & $\begin{array}{l}\text { Higher systolic blood pressure and triglycerides } \\
\text { and lower high-density } \\
\text { lipoprotein (HDL) cholesterol than controls }\end{array}$ \\
\hline $\begin{array}{l}\text { Merz [20] } \\
2016\end{array}$ & Cross-sectional & 25 vs. 270 & $62.6 \pm 11.6$ & $\begin{array}{l}\text { Clinical features of PCOS were not a significant } \\
\text { predictor in prognostic models including } \\
\text { hypertension, diabetes, waist circumference, } \\
\text { and angiographic coronary artery disease }\end{array}$ \\
\hline
\end{tabular}

\section{Results}

Schmidt et al. carried out long-term, prospective follow-up research of 35 postmenopausal women with PCOS and 120 age-matched controls. The population participated in a study in 1987 and was reinvestigated 21 years later. The aim of their study was to examine whether postmenopausal PCOS women differ from the control group with regard to cardiovascular risk factors, myocardial infarction, stroke, and mortality. They studied parameters such as blood pressure, insulin, triglycerides, total cholesterol, and fibrinogen as well as incidences of myocardial infarction, stroke, hypertension, and diabetes. They found that menopausal patients with PCOS had a higher prevalence of hypertension and higher triglyceride levels than controls. The well-described cardiovascular profile in pre- and perimenopausal PCOS women does not entail an evident increase in cardiovascular events during the postmenopausal period [12].

Armeni et al. conducted a study to evaluate the effect of PCOS on markers of subclinical atherosclerosis in nondiabetic postmenopausal women. They examined blood pressure, blood samples, and arterial structure (intima-media thickness, atheromatous plaque presence) and function (flow-mediated dilation, pulse wave velocity, augmentation index) by ultrasound. They obtained similar results to Schmidt's study. Armeni et al. ascertained that postmenopausal women with PCOS had higher systolic blood pressure and triglycerides and lower high-density lipoprotein (HDL) cholesterol than controls. The values of pulse wave velocity differed significantly between PCOS participants and controls. According to the authors, arterial stiffness is increased in asymptomatic, nondiabetic women with PCOS independently of age, BMI, or blood pressure [13].

Another study, carried out in PCOS women of reproductive age, gave evidence that the insulin resistance of arterial endothelial cells is associated with reduced synthesis of nitric oxide and increased synthesis of vasoconstricting agents. This leads to increased vascular stiffness and consequent hypertension. The authors also found elevated levels of tissue plasminogen activator in such patients, which correlated with body mass index (BMI) and insulin resistance [14].

Elting et al. investigated the prevalence of diabetes mellitus, hypertension, and cardiac diseases in a Dutch population with PCOS, comparing these conditions in the healthy Dutch female population. In PCOS women aged $45-54$ years $(n=32)$ the prevalence of hypertension was 2.5 times higher than the corresponding age group of the Dutch female population. The strongest predictor of hypertension was elevated BMI [15].

Wild et al. conducted a retrospective study of women diagnosed with PCOS an average of 31 years previously and found an increased prevalence of hypertension compared to a cohort of control women [16]. Dahlgren et al. studied if the hormonal imbalance in women with polycystic ovary syndrome continues into and after menopause and analysed factors constituting an increased risk for developing metabolic disorders. Blood pressure was examined in menopausal women with PCOS, who had undergone ovarian wedge resection. It was a long-term follow-up study that showed that menopausal women post ovarian wedge resection were three times more likely to have hypertension compared to non-PCOS women [17].

Most of the available literature data suggest an increased prevalence of elevated blood pressure in 
women with PCOS compared to the healthy population. However, obesity is also a factor complicating the interpretation of these studies, as a well-known risk factor for hypertension [18].

Dalmasso et al. tried to ascertain whether chronic hyperandrogenaemia would result in a more harmful effect on metabolic function, blood pressure, and renal function than normo-androgenaemic age-matched females. They applied an animal model using rats. Female rats were implanted with dihydrotestosterone and were studied at 13 months. In this group it was observed that their blood pressure was significantly higher, heart rate was lower, and renal function (GFR) was reduced by $40 \%$. They also observed that insulin resistance was more significant in rats with higher plasma insulin, normal fasting blood glucose, abnormal oral glucose tolerance test, and higher non-fasting blood glucose. Thus, the chronically hyperandrogenic female rat is a model of postmenopausal PCOS, which exhibits insulin resistance and visceral obesity, hypertension, and debility in renal function [19].

On the other hand, Merz et al. claimed that clinical features of PCOS were not significant predictors in prognostic models for hypertension, diabetes, waist circumference, and angiographic coronary artery disease. However, these data should be interpreted with caution because of the small cohort of participants (295 postmenopausal women, only $25 \%$ with clinical features of PCOS defined by a premenopausal history of irregular menses and current biochemical evidence of hyperandrogenaemia). Their findings question whether identification of clinical features of PCOS in postmenopausal women who already have known cardiovascular disease provides any additional opportunity for risk factor intervention [20].

\section{Discussion}

Why are postmenopausal women with a history of PCOS more prone to develop hypertension compared to postmenopausal non-PCOS women?

Generally, long-term risk of cardiovascular complications has been linked to an increase in oxidative stress and inflammatory markers.

Some studies have shown that in women with PCOS endothelial dysfunction goes together with, and is influenced by, the presence of increased serum levels of cytokines and endothelial activation markers. These parameters appear to be correlated with hyperandrogenaemia in this insulin-resistant population [21].

Endothelial dysfunction is the starting point of developing cardiovascular disease [22].

Hypertension develops because of decreased vascular compliance and endothelial dysfunction in women with PCOS [23]. Endothelial dysfunction is proportional to the level of androgens and insulin resistance [21].
Hyperinsulinaemia influences the hypertrophic effect on vascular endothelial and smooth muscle cells; it also advocates endothelin 1 (ET-1) production [23]. ET-1 is a vasoactive substance involved in regulating endothelial function, which is commonly chronically increased in women with PCOS. ET-1 is a product of oxidative stress and also a marker of endothelial dysfunction [24].

It has also been proven that androgens may influence microcirculatory regulation with effects on both vascular smooth muscle and the endothelium through actions on substances in the blood such as endothelin 1 [24].

Moreover, intensification of expression of sICAM-1 and SVCAM-1 molecules was observed in PCOS women, which play an important role in focal leukocyte accumulation in subendothelial regions of atheroma [21, 25]. In addition, there is correlation between SVCAM-1 and hyperandrogenaemia. High testosterone levels have been found to induce VCAM expression. Androgens might promote monocyte adhesion to endothelial cells in the development of atherosclerosis [25]. A proatherogenic process might be indicated in PCOS-women by extended CRP levels - another cardiovascular risk factor - and endothelial dysfunction [26]. Data suggest that increased levels of CRP are consistent with the severity of endothelial dysfunction, which supports the hypothesis that chronic inflammation contributes to endothelial dysfunction [21]. Elevated serum levels of ET-1 have been shown to cooperate with other markers of endothelial activation, endothelial dysfunction, and low-grade chronic inflammation molecules. It was found that there is a relationship between ET-1 and hyperandrogenaemia [27].

Advanced glycation end products (AGEs) are pro-inflammatory molecules that trigger a state of intracellular oxidative stress and inflammation after binding to their cell membrane receptors RAGE [28]. In women with PCOS, the concentration of AGEs is increased, and it has a pathogenic significance in many complications. Reason for pathological changes of AGEs include activating oxidative stress and inflammation [29]. PCOS women have elevated serum/ovarian AGEs and ovarian RAGE women with PCOS have a systemic chronic inflammatory condition even at the ovarian level, as represented by elevated levels of serum/ovarian AGEs and increased expression of the pro-inflammatory RAGE in ovarian tissue [30].

It has also been found that the insulin resistance of the arterial endothelial cells is associated with decreased synthesis of nitric oxide and increased synthesis of vasoconstricting agents. This leads to increased vascular stiffness [14].

Endothelium dysfunction occurs at the beginning of the atherosclerotic process [31]. Measuring carotid intima-media thickness is a non-invasive method for diagnosing atherosclerosis [32]. Carotid intima-media 
thickness is a structural change in the vascular system visualised by ultrasonography, and it is a strong predictor of cardiovascular events [33]. According to Armeni et al., arterial stiffness is increased in women with PCOS. It might be a mechanism through which PCOS increases the risk of CVD and hypertension [13].

In other research, which was conducted on young women with PCOS, early markers of atherosclerosis were studied using CVD markers assessed by ultrasonography, including: common carotid artery stiffness index and distensibility, and intima-media thickness. It was found that already in young age, women with PCOS exhibit changes in vascular elasticity even in the absence of classical risk factors for CVD, such as hypertension and obesity [34]. Regarding the contribution of individual PCOS features, hyperandrogenism and insulin resistance are the most significant predictors of arterial stiffness [13]. An attempt to explain the effects of hyperandrogenism on arterial stiffness is based on rats model - testosterone has influence on vascular contraction and interferes with oestradiol-mediated endothelium-dependent vasodilation [35]. Central arterial stiffness is characterised by excessive collagen in the vessel wall, an amount higher than the presence of elastin, induced by the inflammatory state and the increase in luminal pressure. Metal-proteinases also play an important role in degrading the matrix protein. Moreover, the glycation phenomenon makes collagen more rigid, reduces elastin, and stimulates inflammation in vessels [36].

Hyperandrogenaemia in women with PCOS is associated with an elevated blood pressure independently of the patient's age, insulin resistance, obesity, and dyslipidaemia [37]. Furthermore, Markopoulos et al. performed a study to examine hyperandrogenism in PCOS after menopause. They found that postmenopausal PCOS women are exposed to higher adrenal and ovarian androgen levels than non-PCOS women [38]. Puurunen et al., based on the results of cross-sectional research, claimed that impaired glucose metabolism, increase ovarian androgen secretion, and chronic inflammation observed in premenopausal women with PCOS continue after their menopause [39]. In conclusion, hyperandrogenaemia might increase the risk of hypertension in postmenopausal women with PCOS.

Ethinylestradiol in combined hormonal contraceptives elevates the production of hepatic angiotensinogen, which causes an elevation of blood pressure via the rennin-angiotensin-aldosterone system, regardless of the route of administration [40]. Despite the evolution of new progestogens, only drospirenone has the antimineralocorticoid activity of endogenous progesterone, regardless of the ethinylestradiol dose present in hormonal contraceptives [41]. Therefore, systolic arterial hypertension is considered an unfavourable effect of contraceptives. That is why the use of hor- monal contraceptives is not indicated for women with hypertension with or without PCOS, regardless of the route of administration or oestrogen type [42]. In the other hand, in another study, it was found that lowering androgen levels with a combined oral contraceptive resulted in lower arterial stiffness over a period of 12 months in women with PCOS [43]. In postmenopausal women, oestrogen replacement therapy ameliorated hyperandrogenicity, and this was accompanied by marked improvements in glucose homeostasis and lipoprotein profile [44]. In another study, women who took hormone replacement therapy had higher SHBG, and lower FAl levels were noted among postmenopausal women who developed CVD events [45].

\section{Conclusions}

Available studies show that women with PCOS more frequently present features of metabolic syndrome and have increased cardiovascular risk factors, including hypertension. Patients with this syndrome should be screened for these civilisation diseases and educated about the lifestyle modifications that could prevent hypertension later in life [46, 47].

Further studies should broaden the knowledge concerning dependency between PCOS, hypertension, and other civilisation diseases.

\section{Disclosure}

The authors report no conflict of interest.

\section{References}

1. Cooney LG, Dokras A. Beyond fertility: polycystic ovary syndrome and long-term health. Fertil Steril 2018; 110: 794-809.

2. Bozdag G, Mumusoglu S, Zengin D, et al. The prevalence and phenotypic features of polycystic ovary syndrome: a systematic review and metaanalysis. Hum Reprod 2016; 31: 2841-2855.

3. Barthelmess EK, Naz RK. Polycystic ovary syndrome: current status and future perspective. Front Biosci (Elite Ed) 2014; 6: 104-119.

4. Lizneva D, Suturina L, Walker W, et al. Criteria, prevalence, and phenotypes of polycystic ovary syndrome. Fertil Steril 2016; 106: 6-15.

5. Ortiz-Flores AE, Luque-Ramírez M, Escobar-Morreale HF. Polycystic ovary syndrome in adult women. Med Clin (Barc) 2019; pii: S00257753(18)30747-4.

6. Behboudi-Gandevani S, Ramezani Tehrani F, Hosseinpanah F, et al. Cardiometabolic risks in polycystic ovary syndrome: long-term population-based follow-up study. Fertil Steril 2018; 110: 1377-1386.

7. Welt CK, Carmina E. Lifecycle of polycystic ovary syndrome (PCOS): from in utero to menopause. J Clin Endocrinol Metab 2013; 98: 4629-4638.

8. Cho LW, Randeva HS, Atkin SL. Cardiometabolic aspects of polycystic ovarian syndrome. Vasc Health Risk Manag 2007; 3: 55-63.

9. Sirmans SM, Pate KA. Epidemiology, diagnosis, and management of polycystic ovary syndrome. Clin Epidemiol 2013; 18: 1-13.

10. Moran LJ, Misso ML, Wild RA, Norman RJ. Impaired glucose tolerance, type 2 diabetes and metabolic syndrome in polycystic ovary syndrome: a systematic review and meta-analysis. Hum Reprod Update 2010; 16 : 347-363. 
11. Behboudi-Gandevani S, Amiri M, Bidhendi Yarandi R, et al. The risk of metabolic syndrome in polycystic ovary syndrome: A systematic review and meta-analysis. Clin Endocrinol (Oxf) 2018; 88: 169-184.

12. Schmidt J, Landin-Wilhelmsen K, Brännström M, Dahlgren E. Cardiovascular Disease and Risk Factors in PCOS Women of Postmenopausal Age A 21-Year Controlled Follow-Up Study. J Clin Endocrinol Metab 2011; 96: 3794-3803.

13. Armeni E, Stamatelopoulos K, Rizos D, at al. Arterial stiffness is increased in asymptomatic nondiabetic postmenopausal women with a polycystic ovary syndrome phenotype. J Hypertens 2013; 31: 1998-2004.

14. Kelly CJ, Speirs A, Gould GW, et al. Altered vascular function in young women with polycysticovary syndrome. J Clin Endocrinol Metab 2002; 87: 742-746.

15. Elting MW, Korsen TJ, Bezemer PD, Schoemaker J. Prevalence of diabetes mellitus, hypertension and cardiac complaints in a follow-up study a Dutch PCOS population. Hum Reprod 2001; 16: 556-560.

16. Wild S, Pierpoint T, Jacobs H, McKeigue P. Long-term consequences of polycystic ovary syndrome: results of a 31 year follow-up study. Hum Fertil (Camb) 2000; 3: 101-105.

17. Dahlgren E, Johansson S, Lindstedt G, et al. Women with polycystic ovary syndrome wedge resected in 1956 to 1965: a long-term followupfocusing on natural history and circulating hormones. Fertil Steril 1992; 57: 505-513.

18. Bentley-Lewis R, Seely E, Dunaif A. Ovarian hypertension: polycystic ovary syndrome. Endocrinol Metab Clin North Am 2011; 40: 433-449.

19. Dalmasso C, Maranon R, Patil C, et al. Cardiometabolic Effects of Chronic Hyperandrogenemia in a New Model of Postmenopausal Polycystic Ovary Syndrome. Endocrinology 2016; 157: 2920-2927.

20. Merz CN, Shaw LJ, Azziz R, et al. Cardiovascular Disease and 10-Year Mortality in Postmenopausal Women with Clinical Features of Polycystic Ovary Syndrome. J Womens Health (Larchmt) 2016; 25: 875-881.

21. Diamanti-Kandarakis E, Alexandraki K, Piperi C, et al. Inflammatory and endothelial markers in women with polycystic ovary syndrome. Eur J Clin Invest 2006; 36: 691-697.

22. Gunning MN, Fauser BCJM. Are women with polycystic ovary syndrome at increased cardiovascular disease risk later in life? Climacteric 2017; 20: 222-227.

23. Shah D, Rasool S. Polycystic ovary syndrome and metabolic syndrome: the worrisome twosome? Climacteric 2016; 19: 7-16.

24. Wenner MM, Taylor HS, Stachenfeld NS. Androgens influence microvascular dilation in PCOS through ET-A and ET-B receptors. Am J Physiol Endocrinol Metab 2013; 305: E818-825.

25. Davies MJ, Gordon JL, Gearing AJ, et al. The expression of the adhesion molecules ICAM-1, VCAM-1, PECAM, and E- selectin in human atherosclerosis. J Pathol 1993; 171: 223-229.

26. Kelly CC, Lyall H, Petrie JR, et al. Low grade chronic inflammation in women with polycystic ovarian syndrome. J Clin Endocrinol Metab 2001; 86: 2453-2455.

27. Diamanti-Kandarakis E, Spina G, Kouli C, Migdalis I. Increased endothelin-1 levels in women with polycystic ovary syndrome and the beneficial effect of metformin therapy. J Clin Endocrinol Metab 2001; 86: 46664673.

28. Pertynska-Marczewska M, Diamanti-Kandarakis E, Zhang J, at al. Advanced glycation end products: A link between metabolic and endothelial dysfunction in polycystic ovary syndrome? Metabolism 2015; 64: 1564-1573.

29. Nayaker BS, Thomas S, Ramachandran S, at al. Polycystic ovarian syndrome-associated cardiovascular complications: An overview of the association between the biochemical markers and potential strategies for their prevention and elimination. Diabetes Metab Syndr 2017; 11 Suppl 2: S841-S851.

30. Diamanti-Kandarakis E, Katsikis I, Piperi C, et al. Increased serum advanced glycation end products is a distinct finding in lean women with polycystic ovary syndrome (PCOS). Clin Endocrinol (Oxf) 2008; 69: 634641.

31. Davignon J, Ganz P. Role of endothelial dysfunction in atherosclerosis. Circulation 2004; 109 (Suppl 1): III27-32.

32. Kaya MG, Yildirim S, Calapkorur B, et al. Metformin improves endothelial function and carotid intima media thickness in patients with PCOS. Gynecol Endocrinol 2015; 31: 401-405.
33. Lorenz MW, Markus HS, Bots ML, et al. Prediction of clinical cardiovascular events with carotid intima-media thickness: a systematic review and meta-analysis. Circulation 2007; 115: 459-467.

34. Soares GM, Vieira CS, Martins WP, et al. Increased arterial stiffness in nonobese women with polycystic ovary syndrome (PCOS) without comorbidities: one more characteristic inherent to the syndrome? Clin Endocrinol (Oxf) 2009; 71: 406-411.

35. Hillebrand $U$, Hausberg $M$, Lang D, et al. How steroid hormones act on the endothelium - insights by atomic force microscopy. Pflugers Arch 2008; 456: 51-60.

36. Scicchitano P, Dentamaro I, Carbonara R, at al. Cardiovascular Risk in Women With PCOS. Int J Endocrinol Metab 2012; 10: 611-618.

37. Chen MJ, Yang WS, Yang JH, et al. Relationship between androgen levels and blood pressure in young women with polycystic ovary syndrome. Hypertension 2007; 49: 1442-1447.

38. Markopoulos MC, Rizos D, Valsamakis G, et al. Hyperandrogenism in women with polycystic ovary syndrome persists after menopause. J Clin Endocrinol Metab 2011; 96: 623-631.

39. Puurunen J, Piltonen T, Morin-Papunen L, et al. Unfavorable hormonal, metabolic, and inflammatory alterations persist after menopause in women with PCOS. J Clin Endocrinol Metab 2011; 96: 1827-1834.

40. Oelkers WK. Effects of estrogens and progestogens on the renin-aldosterone system and blood pressure. Steroids 1996; 61: 166-171.

41. Sitruk-Ware R. New progestagens for contraceptive use. Hum Reprod Update 2006; 12: 169-178.

42. de Melo AS, Dos Reis RM, Ferriani R, at al. Hormonal contraception in women with polycystic ovary syndrome: choices, challenges, and noncontraceptive benefits. Open Access J Contracept 2017; 8: 13-23.

43. Vieira CS, Martins WP, Fernandes JB, et al. The effects of $2 \mathrm{mg}$ chlormadinone acetate/30 mcg ethinylestradiol, alone or combined with spironolactone, on cardiovascular risk markers in women with polycystic ovary syndrome. Contraception 2012; 86: 268-275.

44. Andersson B, Mattsson LA, Hahn L, et al. Estrogen replacement therapy decreases hyperandrogenicity and improves glucose homeostasis and plasma lipids in postmenopausal women with noninsulin-dependent diabetes mellitus. J Clin Endocrinol Metab 1997; 82: 638-643.

45. Rexrode KM, Manson JE, Lee IM, et. al. Sex hormone of cardiovascular events in postmenopausal women. Circulation. 2003; 108: 1688-1693.

46. Ireland K, Child T. Polycystic ovary syndrome and the postmenopausal woman. J Br Menopause Soc 2006; 12: 143-148.

47. Shah D, Bansal S. Polycystic ovaries - beyond menopause. Climacteric 2014; 17: 109-115. 